\title{
The Modern Changes of “Gesar” Thangka Under Multiple
}

\section{Contexts}

\author{
CHEN Meizhen \\ Southwest Minzu University, Chengdu, China
}

\begin{abstract}
“Gesar” Thangka is an important type of Tibetan Tangka. With the generation and spread of Gesar epic, “Gesar” Thangka is also undergoing complex historical changes in the social and historical process of Tibetan areas. Since the 1980s, in the context of the rescue of Gesar epic, the rise of market economy, and the protection of intangible cultural heritage, “Gesar” Thangkar has gained creative vitality and experienced modern changes, presenting the aesthetic characteristics of secularization, sanctification, and art.
\end{abstract}

Keywords: “Gesar” Thangka, multiple context, modern alteration

\section{Introduction}

"Gesar” Tangka is an image presentation of the Tibetan epic "Gesar”, known as "Zhong Tang”. Compared with other types of Thangka, Zhong Tang has more freedom in creation. As a result, "Gesar" Thangka, accompanied by the changes of social history and its living circumstances, has also undergone complex historical changes in the pursuit of aesthetics. Since the 1980s, when Gesar epic was rescued and then is known as non-material cultural heritage "Gesar" Tangka regained vitality, it began to appear in the cultural institutions, temples, Buddhist halls, gallery market, and other cultural backgrounds. As a result, "Gesar” Thangka shows the mixture aesthetic characteristics of secularization, sacredness, and artistry.

\section{The Emergence and Development of "Gesar" Thangka}

"Gesar" is a heroic epic that has been spread in the Tibetan area of China for more than 1,000 years. At present, it is generally believed that the legend of Gesar began to circulate in the period of the Tubo Dynasty. By the later 8th century, King Gesar and related legends appeared in the Tibetan literature, which can determine the story of Gesar began to be spread in Tibetan folk culture during Tubo Dynasty.

For thousands of years, Gesar has been handed down mainly by the rap of folk artists. In order to enhance the effect of rap, and to attract and serve more listeners, some clever folk artists use "Gesar" as the theme, drawing figure paintings and Gesar stories, hanging up while rap, explaining while singing. This is the Tangka painting about Gesar, and over time it became a special type of Thangka_Zhong Tang. (Jiangbian Jiacuo \& Zhou, 2003, p. 126)

Fund project: This paper is a general art project of the National Social Science Foundation in 2017: stage achievement of "The Study of 'Gesar' Thangka in the Inheritance of Tibetan Epic Culture” (Project Number 17BH153); the stage achievements of 2017 National Social Science Foundation major project "Investigation and Research on Image Culture of Hero Epic 'Gesar' and Database Construction” (Project Number 17ZDA160).

CHEN Mei-zhen, Associate Professor, Art College, Southwest Minzu University, Chengdu, China. 
Due to historical reasons, the earliest works of "Gesar" Thangka at present are works of Ming Dynasty, so the development status of "Gesar" Thangka in a long period of history is not known to us due to the lack of image, text, and documentation records. However, from the works of "Gesar" Thangka in the Ming Dynasty and Qing Dynasty, we can clearly know that "Gesar" Thangka has developed from a mere auxiliary of rap to a sacred offering with religious attributes along with the course of history. Most of the "Gesar” Thangka from the Ming and Qing Dynasties that can be seen today are worshipped in temples, for example, the "Gesar horse-riding battle statue” (Ming) in Yiniu Temple, Shiqu County, Ganzi Tibetan Autonomous Prefecture, Sichuan Province, the "Gesar riding-horse battle statue" (Ming) in the Xiangkha Temple in Batang County, and the "Gesar King and his Lingguo generals” in the Donggu Temple of Ganzi County (Qing), “Gesar horse-riding battle statue” (Qing) in Zongsa Temple, Dege County, "Gesar and the gods of the mountains” (Qing) Gongqing Temple, Dege County (Feng, 2014, pp. 200-202). Until the early 20th century, many scholars at home and abroad could see temples and ordinary houses dedicated to "Gesar" Tangka when they visited Tibetan areas.

Among the people, “Gesar” Thangka, especially Gesar riding-horse Thangka, is worshiped and enshrined, not only as an artistic work, but more as the picture of Warrior. In the past, when soldiers were ready to go out for battle, they hung up "Gesar riding-horse Thangka” to burn incense and pray for the protection of the Warrior to make them victorious. (Xu, 1993, p. 57)

The appearance of "Gesar" Thangka as a religious relic is closely related to the spread and development of Gesar's rap. According some Tibetan documents, it can be found from Tibetan documents that Gesar's story has been obviously influenced by Buddhism at least in the 16th century. Later, a group of monks and scholars in Tibetan areas transformed and utilized the content of the story of Gesar. Among them, the famous Tibetan scholar, the living Buddha of Juga Temple, Cumeiban Ranggigatso, when he wrote the Gesar story, he transformed it into the "Gesar King Biography” with the content of Buddhism, and transformed Gesar into a Buddhist dharma god. Besides him, Tibetan Buddhism Nyingma sent many of the monks to write the eulogy of this type; part of the Gelug monks also has the relaed work. For example, the living Buddha of Ganzi Temple in Ganzi County wrote “Gesar Prayer”, another eminent monk in Kang District, Reshe Duojie Dorje, wrote "Gesar Prayer”, “Gesar Achievement Law”, and so on. In this connection, King Gesar was gradually deified by Tibetan Buddhism from secular people to the gods of Tibetan Buddhism, and was also considered to be the embodiment of Manjushri Bodhisattva, the angel of vajra, the hand of padmasambhava, and so on. Xu Guoqiong described the image of Gesar that he knew in Tibetan areas in his works.

Gesar is generally worshiped as a god. Some say that he is Warrior, war to worshiped him; some said he was the god of wealth, praying that cattle and sheep would flourish and accumulate wealth and treasure, and people would worship him in peacetime; some said he was a local god, saying that where he was worshiped, people and animals would be safe and happy. In a word, he was venerated as a god. (Qingcuo, 2013, p. 81)

As mentioned above, it is also very obvious that Gesar was depicted as a god in the "Gesar” Thangka of the Ming and Qing Dynasties. "Gesar Riding-Horse Statue in the Battle” Tangka and Buddhist Tangka have the same characteristics in composition, strictly following the Thangka painting measure scale of the classic book, "the Buddha said the Statue Metrics” to create; the main image at the center of the picture is Gesar's horse-riding statue. In the specific details, in order to pursue the symbolization of the god of war, King Gesar sits sideways on the horse, and the whole shape is completely out of proportion. Instead, he made every effort to maximize the 
integration of King Gesar and the Horse. The same pursuit can be seen in color processing Gesar's lower body harmoniously aligned with the colors of the Horse. For example, the "Gesar Riding-Horse Statue in the Battle" wihch is now hidden in of the Research Institute of Qinghai Federation of Literature and Art and collected by the Qinghai Folk Literature Collection team in the 1950s, the age of Tangka creation, is considered to be the Ming Dynasty. In this Thangka work, Gesar sat on horseback, with the red of the lower body so close to the horse's color that it took great care to find the left leg on which King Gesar stepped on his foot. Secondly, the battle statue itself is a dynamic shape, but the painters, when dealing with it, held it down very well, as far as King Gesar's style is concerned, the slight tilt angle of the upper and lower halves and the lift of the whip’s right hand have an upward momentum, but his gazing at the earth and the downward pressure of their left hand with spear stifle the upward movement. The hoofed position of the horse in the crotch seemed to emphasize the movement of running, but at the same time with a low head to suppress the movement, so that the image of the god of war has a solemn feeling, like the silence of the Buddha, with a permanent. For the providers, the holy images in the "Gesar" Thangka paintings are the objects of their meditation and worship, which are cautiously hung on the walls or beams of the family Buddhist hall and temples.

The transformation of function and identity of the "Gesar" Thangka from its emergence to development is closely related to its image presentation as Gesar's epic of rapping Gesar. Therefore, since the 1980s, in the context of the rescue and protection of Gesar's rap, the transmission of Gesar's epic and the formation of Gesar's epic culture, the "Gesar" Thangka has also undergone .

\section{Modern Changes of “Gesar” Thangka}

After the prosperity of the Ming and Qing Dynasties, the creation of "Gesar" Thangka was depressed due to historical reasons. In the 1980s, the rescue work of gesar's epic poetry made "Gesar” Thangka's creation regain artistic life; many "Gesar" Thangka are created in this period, such as the giant painting scroll "The Grand View of Chinese Tibetan Culture and Art Painting” in Qinghai Province, 1,000 Gesar Thangka Paintings in Sichuan Province, Gesar 30 Senior Generals in Tibetan academy of social sciences. In the 21st century, the Gesar epic has been identified as a national and world non-material cultural heritage, people in rescue Gesar, rap artist, collect Gesar epic of the text at the same time, also started the massive "Gesar" Thangka creation. Under the multiple context "Gesar" Thangka underwent functional changes, and finally his works presented the aesthetic characteristics of secularization, sanctification, and artistry.

\section{Function Changes of "Gesar" Thangka}

Although "Gesar" Thangka was widely spread in Tibetan areas from the Ming and Qing Dynasties until the early 20th century, due to complex historical reasons, it gradually faded out of people's sight after the 1960s in Tibetan areas. It was not until the 1980s that the rescue work of the epic "Gesar" made the creation of "Gesar" Thangka regain the artistic life. Renqing Jiancuo’s creation of "Gesar” Thangka in Jianza County, Huangnan Tibetan Autonomous Prefecture, Qinghai Province, started mainly from the "Gesar Art Rescue Office”, which was established in 1979. In 1980, he wrote "Ling·Gesar King and the Thirtieth General” published in the Tibetan periodicals "Zhangchal” and "the Art of the masses of Qinghai". In the 1990s, when the rescue and investigation results of Gesar's epics were published successively, the creation of "Gesar" Thangka entered the period of the creation of a series of works. In 1996, when the Chinese Academy of Social Sciences organized and published $A$ 
Selectiong of Gesar, it hired some folk painters from Huangnan Tibetan Autonomous Prefecture of Qinghai Province to draw 240 paintings of Gesar Thangka. In 1998, Renqing Jiancuo's Thangka works on the theme of “Gesar” epic, "Hawling War”, "Horse race king”, “Sindan Tiger Lion confrontation”, “Gesar Blessing Ode”, "Demonic Chapter”, "Shadowline Chardonnay”, "The Birthday of the Ridge”, and "The World Mulberry” are collected by the Gesar Research Center of the Chinese Academy of Social Sciences. In 1997, Genqiu Tashi, a native of Dege in Sichuan Province, started the painting project of 1,000 "Gesar” Thangka paintings, and tried hard to express a complete story of Gesar in each painting, and finally formed the world's first biography of King Gesar in the form of Thangka. In the 21st century, Gesar's epic poem was successively listed on the national and world-class intangible cultural heritage. The government of Ganzi Tibetan Autonomous Prefecture , Sichuan Province also joined the project of 1,000 Gesar's Thangka Paintings. Later, in order to commemorate the millennium activities of gesar epic, the Tibetan Academy of Social Sciences led a group of Thangka painting artists to draw a set of 21 paintings of Gesar Thangka in 2002. The town of Jiegu in Yushu Tibetan Autonomous Prefecturehas built a special Gesar cultural exhibition hall in the Gesar Cultural Square rebuilt after the earthquake. More than 100 pieces of the Gesar Thangka are hung in the exhibition hall. In the activity of "Gesar Entering Campus”, Yushu Second Nationality Senior High School employed painters and students from Thangka class of the school to create a long scroll of "The Great View of Thangka Painting of Gesar Heroes" by virtue of the school's own strength. It can be seen that since the 1980s, the creation of Thangka's works in Gesar has been inseparable from the context of Gesar's epic culture, which has also brought about changes in the production field of the Thangka. The collections and displays of public cultural space such as exhibition halls and museums have made the Gesar Thangka an important way of spreading and inheriting the culture of Gesar.

\section{Aesthetic Transmutation of “Gesar” THangka}

The change of the living field and function of "Gesar" Thangka finally led to the change of the artists' aesthetic pursuit. In the end, Gesar Tangka’s works presented the characteristics of secularity, sacredness, and artistic mixture.

Traditional Thangka works have clear measurement requirements and central dominant composition characteristics, but since the new era, in addition to inheriting the traditional Thangka central composition, more innovative works have appeared. The composition of "1,000 Gesar Thangka Paintings" is the composition of comic strips. Each work is based on the content of the story, and the image of Gesar is not placed in the center of the picture as the main character.

The Tangka story map of Gesar discovered at Senyong Art Palace in Dari County; Guolo Tibetan Autonomous Prefecture is also different from that of Gesar in Qing Dynasty. There is still no central figure in the story, but the story scene is arranged from top to bottom according to the vertical axis of Tangka. In the "Volume of Seizing the Silk the Milwaud Kingdom” for the selected illustrations of "Gesar", Tangka even adopted a close-up composition; the whole central part of the picture is occupied by a huge fish head. From the perspective of character shaping, both the figure painting and the story painting of "Gesar" emphasize the authenticity and secularization of the characters. The proportion of the human body is closer to that of the normal people. Faces, hands, and other parts that express muscle reflect the relationship between light and dark with the transition of color, showing a sense of three dimensions. The figure facial expression molds exquisite vivid, each kind of 
character characteristic all manifests incisively and vividly. For example, teacher Tudeng Weise, in Banma County, placed in his studio a picture of the figure "Gesar Riding Horse Statue in Battle", which was about to be finished, with delicate depictions. Although the facial expression is mainly flat smear, in the color rendering around the eyes of the characters and the corners of the mouth, it is obvious that the colors are used to highlight the three-dimensional feeling of the face. Around the image of the Thirteen Wilmal is lifelike, and it possesses the form and spirit, in the treatment of hair, with the use of traditional Chinese painting "hair tearing" technique to strengthen the texture, so that these places are bright and the schemata are like the highlights in an oil painting. In terms of space performance, in this period, "Gesar" Thangka began to pay attention to the perspective relationship and to pursue the reproduction of space. The "Gesar' Thangka of Thousands of Pictures” uses the scattered point perspective of Chinese painting to deal with the background and integrate the ethereal world of epic legends into real life. Each story shows as much as possible the scene that really happens in real life. The picture shows mountains, grasslands, rivers, cattle, sheep, tents, and other familiar and friendly natural environment.

In addition, an important feature of "Gesar" Thangka's creation in this period is that it is no longer the “nameless creator”, but the artist’s distinctive and individualized creation. Renqing Jiancuo's “Gesar” creation has a clear personal stylized characteristic from beginning to end. In his 2008 composition of the "Ling.King of Gesar and the Seven Major Generals”, in color, it gives up the traditional Thangka technique of using bright colors and light and thin flat painting, and with the red and black two colors to highlight the thick feeling of color, so as to achieve the majestic force and the air of justice that highlight the image of the characters. The whole work has a kind of elegance and dignity of classical oil painting. In the Gesar Cultural Exhibition Hall of Jiegu Town, Yushu prefecture, the influence of modern meticulous painting can be clearly seen in the creation of "Gesar" Thangka, especially in the portrayal of female images, with a total of about 30 female images depicted in the whole venue, which includes the Tibetan, Mongolian, Miao, Uygur, and other ethnic women who are involved in the female image; all women are slender and slim, posture or sitting or standing, beautiful and moving; the dress on the body depicts exquisite; all line sketches are fine smart. Of course, the artist's personal emphasis can also be seen from the signatures of artists in Thangka's works. In the Gesar Culture Exhibition Museum of Jiegu Town, Yushu prefecture, two large paintings of "Gesar" are painted on the lower right rock of the picture with the bright and orderly seal of Thangka painter Quxiong Zeren. When the author visited Banma County, the author found that Tudeng Weise had signed his name with small strokes in his works.

In general, the works of "Gesar" Thangka since the 1980s have a stronger artistic pursuit on the basis of their secularization and sanctification. More emphasis is placed on the authenticity of the characters, the life of the scene and the individualization of the techniques of expression in artistic expression. As a result, these pictures of "Gesar" Thangka are particularly delicate, showing the artistic pursuit of Tangka painters and the inclusiveness of Gesar's culture.

\section{Conclusion}

The modern changes of Gesar Thangka’s creation does not only show the variation of "Gesar" epic in the course of historical change in Tibetan area, at the same time, we can see the cultural choices made by the Tibetan cultural elite with the change of the times. Especially since the 1980s, "Gesar" epic culture has become an 
important part of the traditional culture of the Chinese nation. In protecting and inheriting the traditional culture and art of this nation, the Tibetan cultural elites absorb and draw lessons from the foreign culture and art, so that we can see the tolerance of this nation; when the authors came into contact with the artists who drew "Gesar" Thangka in Qinghai field survey, they inherited this artistic form of traditional painting Thangka of their own nationality; they all began to explore their own innovation. As mentioned above, Peng De followed the online video self-taught oil painting, in his studio also hunging a number of oil paintings and water powder works; Tudeng Weise teacher went to Shanghai to study painting twice, and also thought that he had the influence of oil painting in the creation of Thangka, while Mr. Renqing Jiancuo definitely used the color of western classical oil painting to create "Gesar" Thangka. The artistic innovation and aesthetic pursuit they have carried out in the creation of Thangka seem to show us that the Tibetan cultural elite is working hard to transform this "religious sacred object" of Thangka into "national art". As Nyima Tserring says,

Since 1,300 years, the Tibetan people have produced religious paintings as worshipers because of their belief in Tibetan Buddhism. If the art of a nation exists only as a sacrifice, it is easy to lose its vitality. The traditional Tangka takes the primitive religion as the content, unable to convey the fresh cultural information of the new era. Only when the life of the Tibetan people is expressed through the International "language" and through the language of modern painting can more people understand the history of this nation and their present life and spiritual pursuits. (2010.3.06)

\section{References}

Feng, J. C. (2014). Chinese encyclopedia of intangible cultural heritage, epic volume. Beijing: China Literary Union Press. Jiangbian Jiacuo, \& Zhou, A. M. (2003). The Tibetan hero epic “Gesar” Tangka. Beijing: China Fine Arts Press.

Qingcuo. (2013). Tibetan art of “Gesar” Tangka. Qinghai: Qinghai People’s Publishing House.

Xu, G. Q. (1993). The investigation of Gesar. Yunnan: Yunnan People’s Publishing House.

Zhoufang. (2010) .The pure land of spirit painted by nima zeren.China national daily. 2010.3.06 